\title{
Fast two-photon neuronal imaging and control using a spatial light modulator and ruthenium compounds
}

\author{
Darcy S. Peterka*a ${ }^{\mathrm{a}}$, Volodymyr Nikolenko ${ }^{\mathrm{a}}$, Elodie Fino ${ }^{\mathrm{a}}$, Roberto Araya ${ }^{\mathrm{a}}$, Roberto Etchenique ${ }^{\mathrm{b}}$, \\ Rafael Yuste ${ }^{a}$ \\ ${ }^{a}$ Dept of Biological Sciences, Howard Hughes Medical Institute, Columbia University, New York, \\ NY USA 10027; \\ ${ }^{\mathrm{b}}$ Departamento de Química Inorgánica, Analítica y Química Física, INQUIMAE, Facultad de \\ Ciencias Exactas y Naturales, Universidad de Buenos Aires, Pabellón 2, Ciudad Universitaria, \\ Buenos Aires, Argentina C1428EHA
}

\begin{abstract}
We have developed a spatial light modulator (SLM) based microscope that uses diffraction to shape the incoming twophoton laser source to any arbitrary light pattern. This allows the simultaneous imaging or photostimulation of different regions of a sample with three-dimensional precision at high frame rates. Additionally, we have combined this microscope with a new class of two photon active neuromodulators with Ruthenium BiPyridine (RuBi) based cages that offer great flexibility for neuronal control.
\end{abstract}

Keywords: Spatial light modulator, two-photon, uncaging, Ruthenium, neuron, imaging, adaptive optics, microscope

\section{INTRODUCTION}

\subsection{Imaging}

Two-photon microscopy has led to tremendous advances in neuronal imaging. By using the nonlinear absorption of two photons of infrared (IR) or near-infrared (NIR) light, multiphoton microscopies offer lower phototoxicity and improved penetration depth than the single photon microscopies, while still maintaining high spatial resolution. This has allowed the long term imaging of brain tissue, both in vitro and in vivo ${ }^{1}$, as well as detailed studies of the structure and function of neurons and their processes. Until recently, however, two-photon imaging suffered from relatively poor temporal resolution, as the high intensity requirements for efficient excitation prohibit wide field excitation so the laser is focused to a single spot that has to be sequentially raster scanned across the sample. While the physical scanning devices, such as galvanomirrors or acoustooptic deflectors, have characteristic maximal speeds, in many cases, the time required to collect an image is limited not by the devices themselves, but by the necessary pixel dwell times required to generate images with sufficiently high signal-to-noise $(\mathrm{S} / \mathrm{N})$ ratios to be useful. Attempts to shorten the necessary dwell times by increasing the excitation power quickly become limited. First, the excitation power cannot be increased arbitrarily, as too high light levels cause photodamage and bleaching. More fundamentally, fluorophores have a characteristic emission lifetime, which limits the number of excitation-emission cycles per unit time. The signal cannot be made brighter with increased laser power, it is effectively saturated ${ }^{2,3}$.

One obvious strategy for increasing the speed of acquisition is to increase the number of beams scanning across the sample. The beamlets are simultaneously swept over the sample, and the signal is recorded with a position sensitive widefield detector such as a camera or a diode or photomultiplier array. The effective acquisition rate is approximately equal to the single-beam rate multiplied by the number of beamlets. With most implementations, the overall resolution decreases as the emitted fluorescence photons are still scattered, and cannot always be mapped to a particular excitation location. However, for many applications, the resolution is still sufficient for detailed imaging.

*dp2403@columbia.edu; phone 1646 330-4609; http://www.columbia.edu/cu/biology/faculty/yuste/

Photonic Therapeutics and Diagnostics VI, edited by N. Kollias, B. Choi, H. Zeng, R. S. Malek, B. J.-F. Wong, J. F. R. Ilgner,

K. W. Gregory, G. J. Tearney, L. Marcu, H. Hirschberg, S. J. Madsen, A. Mandelis, A. Mahadevan-Jansen, E. D. Jansen, Proc. of SPIE Vol. 7548, 75484P - (C) 2010 SPIE · CCC code: 1605-7422/10/\$18 - doi: 10.1117/12.842606 
Raster imaging schemes suffers from one additional problem. Many samples do not fill the whole image field-of-view (FOV), and as a result, the scanning beams end up spending time illuminating areas between regions of interest (ROI) rather than the ROIs themselves. This "wastes" time and excitation power, the latter being an important consideration for non-linear microscopies. Here we introduce a different, flexible method for increasing the speed of functional imaging; using a programmable spatial light modulator to diffract the incoming beam into multiple beamlets, targeting only the ROIs ${ }^{4}$. The illumination pattern is quasi-static, with all points simultaneously excited, and the resulting fluorescence collected on a wide field detector, such as a CCD.

\subsection{Uncaging}

In addition to merely watching functional activity, the introduction of two-photon active caged neuromodulators allows for the control of neurons with the same subcellular precision. Ideally, these modulators would allow the experimenter direct control over the state of the cell, both excitation and inhibition. Currently, though, no approach works perfectly. The "traditional" two photon photoreleasable cages are based on functionalized nitrobenzyl derivatives, and have proven to be very useful for studying excitatory activity. For example, 4-methoxy-7-nitroindolinyl-amino glutamate ${ }^{5}$ (MNIglut) has been used to activate individual spines, trigger action potentials in cells, and map synaptic receptors ${ }^{6,7,8,9,10}$. Unfortunately, MNI-glut needs to be used at millimolar concentrations for effective two-photon uncaging, and at these concentrations, like many other caged compounds, it is an antagonist of GABAergic transmission ${ }^{11}$. For studies of neuronal circuits, this is a significant problem as it can cause epileptiform events. It also limits the study of inhibitory network activity and connectivity, because it blocks GABAergic responses. Two-photon uncaging studies of GABAergic activity has also been limited by the lack of caged GABAs with sufficiently high two photon cross sections.

To combat these problems, we have utilized the flexibility of ruthenium chemistry, and introduced a series of caged compounds based on ruthenium-bipyridine complexes ${ }^{12}$. These compounds have high one- and two-photon uncaging cross sections and photorelease the bound neuromodulators quickly and cleanly - the initial excited state quickly evolves into a dissociative state that leads to a single metal-ligand bond cleavage, freeing the compound of interest. The compounds also seem to exhibit less nonspecific effects than the currently used nitrobenzyl based compounds ${ }^{11}$. Also, the ruthenium based cage can be applied to a wide range of target compounds, and has been extended to include glutamate, GABA, 4-AP, and others.

\section{METHODS}

\subsection{Slice Preparation and Electrophysiology}

The details of our protocols are described in detail elsewhere, so only a summary is given here ${ }^{4,11}$. Animal handling and experimentation was done according to NIH and local IACUC guidelines. $350 \mu \mathrm{m}$ thick coronal slices from 14 day old C57BL/6 mice were prepared and transferred to the recording chamber on the microscope. The recording chamber was bathed in artificial cerebrospinal fluid (ACSF, $\mathrm{pH} 7.4$ ) kept at room temperature and saturated with $95 \% \mathrm{O}_{2}$ and $5 \% \mathrm{CO}_{2}$. The layer $2 / 3$ and layer 5 cortical pyramidal neurons were whole-cell patched, with $\sim 5 \mathrm{M} \Omega$ glass pipettes, which were filled with artificial intracellular solution.

For the fast functional imaging studies, before transferring to the recording chamber, the slices were bulk loaded by incubation in the dark with a membrane permeable calcium indicator, either Fura-2AM or mag-Indo-1AM. For the uncaging experiments, Alexa 594, an indicator dye, was added to the intracellular solution for visualization of the patched neuron.

\subsection{SLM Microscope}

The detailed construction of our microscope, as well as the optical layout of the instrument has been described in detail elsewhere, so only a brief description of the components is given here ${ }^{4}$. Our microscope consists of a custom modified Olympus BX51 coupled to a diffractive phase-only SLM. We use a high resolution reflective 1080P SLM from Holoeye with phase-only modulation (1920x1080 pixels, 8 -bit phase quantization, with over $2 \pi$ phase modulation at each pixel, $60 \mathrm{~Hz}$ update rate). The surface of the SLM is imaged onto the back aperture of the objective with a system of relay lenses. A pair of galvanomirrors is located at an intermediate image plane, and allows for large scale shifts in the image, as well as traditional raster imaging when the SLM is inactive. The incoming laser beam is expanded to fill the surface 
of the SLM, and the magnification of the intermediate relay telescope serves to scale the beam so that it fills the back aperture of the objective, allowing for diffraction limited performance.

The SLM is able to generate arbitrary illumination patterns on the sample plane by controlling the spatial phase profile of the incoming laser beam. According to the well known Fourier relations in optics ${ }^{13}$, if a transparent object is placed one focal length from the front of a thin lens, the Fourier transform of that object will be formed one focal length behind the lens. This means that we can represent the incoming field at focal $l_{\text {front }}$ by the complex amplitude $E_{k}$, and the field at focal $l_{\text {back }}$ by $F_{k}$, with $E_{k}$, and $F_{k}$ as Fourier transform pairs. The optical path in our microscope is slightly more complex because of a system of relay lenses, but effectively the SLM is located at focal $l_{\text {front }}$, the sample plane at focal $_{\text {back }}$, with the objective acting as the thin lens. Because of this relation, given a particular desired light distribution in the sample plane, it is relatively easy to compute a phase mask for the SLM that will generate it. Though Holoeye provides software that can generate the phase masks, for additional control we sometimes use our own custom developed software routines based on standard iterative-adaptive algorithms ${ }^{14}$. The algorithm starts with the known intensity distribution of the laser, and applies a random phase (aids convergence). The complex field is Fourier transformed, and the resulting intensity distribution compared to the desired one. If the difference exceeds the error tolerance, the amplitude, but not the phase, is modified to better match the desired image. The inverse transform is performed, giving rise to a new input field, and the cycle begins again. The flowchart of the algorithm is shown in figure 1. More complete information on the variety of algorithms can be found in the literature ${ }^{15}$.

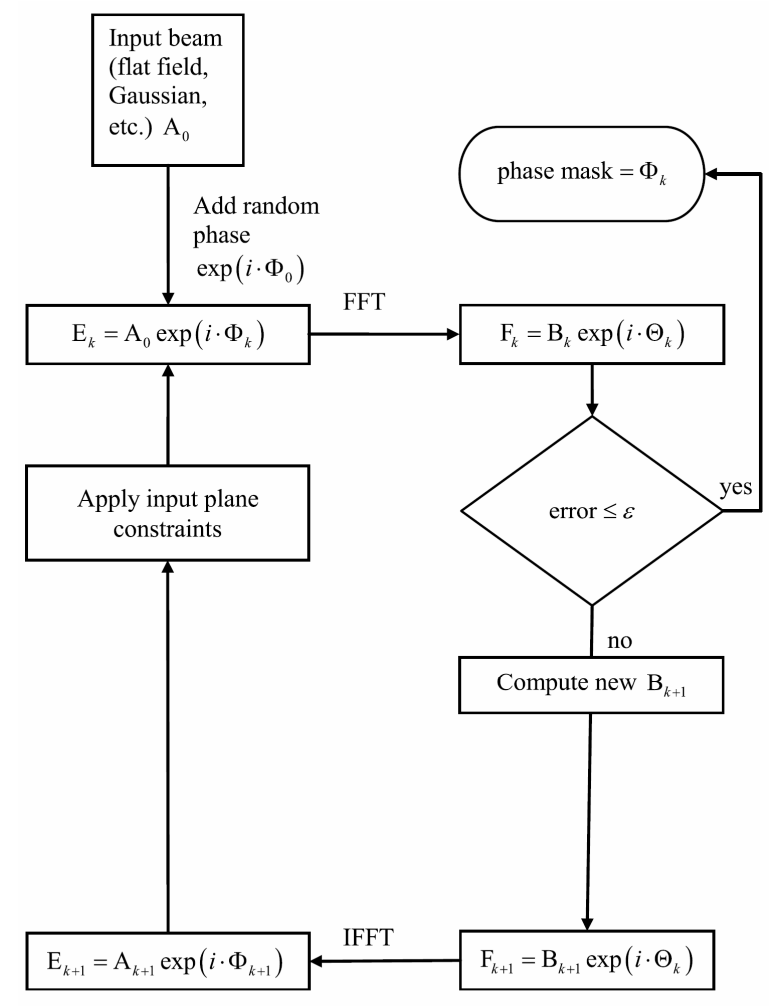

Figure 1. Flowchart of iterative-adaptive algorithm to determine SLM phase mask formation, described in more detail in section 2.2

For experiments, the pattern of interest was generated by first taking a traditional image of the sample, either by traditional two-photon scanning, or by using a calibrated widefield visible light image. Either manual targeting, or an automated segmentation algorithm identified the cell bodies, and identified the centroid of the soma. These were then used as illumination targets. The maximum allowable number to targets is determined by the dividing the total available laser power by the average power needed per ROI. In our experiments with calcium imaging, targeting cell bodies of well loaded cells, we find we can image $\sim 100$ cells simultaneously with sufficient $\mathrm{S} / \mathrm{N}$ to allow for frame rates approaching $30 \mathrm{~Hz}$. In our two-photon uncaging studies, the power required to release enough glutamate to trigger an action potential is significantly higher than that required to acquire an image. As a result, when using the SLM with a 
simple uncaging protocol - a fixed single or double spot on the soma - we could only simultaneously fire a few cells. As described in the next section, more advanced protocols for uncaging, with multiple targets generate better responses. We expect that continuing improvements in the uncaging protocol, by including complex target patterns, along with new developments in chemical cages (or optogenetic methods), will enable us to increase this number in the near future.

\subsection{Uncaging}

Complete details about the two-photon uncaging protocols have been published elsewhere ${ }^{11}$. An aliquot of the desired ruthenium-bipyridine (RuBi) compound (Tocris Bioscience), dissolved in ACSF, was added to the bath, yielding a final concentration of 300-500 $\mu \mathrm{M}$ for the caged compound. The targeted cells were either two photon imaged, with low power, or located under widefield IR illumination. As the RuBi compounds are sensitive to short wavelength visible light, the room was kept dark, and computer screens and other light sources were shielded with red filters (Rosco \#27). For all the two-photon uncaging experiments, the laser was a femtosecond Ti-Saphire (Coherent Chameleon) operating at $800 \mathrm{~nm}$.

Unless otherwise indicated, protocols described below are for uncaging without an SLM. Because of the small twophoton active volume, and hence the small amounts of RuBi available for uncaging, we used a multisport targeting scheme to fire the pyramidal cells reliably. Eight locations were chosen on the soma, and each was illuminated for $8 \mathrm{~ms}$, yielding a total illumination time of $64 \mathrm{~ms}$. The eight targets were themselves "complex" with the actual beam consisting of 5 very closely spaced beamlets generated by a fixed pattern diffractive optical element (DOE). The average laser power on sample was $\sim 200 \mathrm{~mW}$. Using this protocal with RuBi-glutamate, we were able to reliably trigger action potentials in cells. It is likely an alternative illumination pattern could produce firing with less total fluence, but our protocol was sufficient for our experiments. We note that based on the power required per cell to induce firing with this two-photon protocol, and our total power budget, we expect that we will be able to fire about 5 or 6 cells simultaneously with the SLM. For subthreshold stimulations near spines, a single laser spot was used, with much less laser power and time $-5 \mathrm{~ms}$ and $20 \mathrm{~mW}$ was sufficient. Multiple simultaneous subthreshold stimulations were possible by using the SLM to target multiple spines. Experiments involving RuBi-GABA required similar powers to produce responses in the cell, with the exception, of course, being that GABA yields inhibitory currents, and doesn't cause the cells to fire.

\section{RESULTS AND DISCUSSION}

\subsection{Multiplexing and imaging with SLMs}

We developed our system from as an extension of an earlier version of our microscope that used fixed DOEs ${ }^{10}$. While DOEs split the beam, they do so with a fixed pattern and are not suitable for targeted imaging. SLMs, on the other hand, are essentially computer controlled, programmable analogues of fixed DOEs, where the beam can be dynamically adjusted to generate the appropriate number of beamlets directed to the correct locations. Digital micromirror devices (DMD) could also be used to dynamically generate complex illumination patterns, but they have one significant disadvantage. DMDs create the patterned illumination by removing light, essentially acting as a physical mask. With the phase-only SLM, the pattern is made by redirecting light to the desired locations. Because the amplitude of the incoming light beam is unchanged, a much larger fraction of the input laser power is available for non-linear excitation. One seeming disadvantage is that the phase-only SLM relies on the coherence properties of a laser, but this is a not an issue for non-linear microscopies, as the high intensity requirements make a laser source mandatory. Because the SLM can imprint almost any phase pattern on the incoming light field, it can also be used to mimic other optical elements, like lenses and prisms. The SLM can correct for aberrations in the optical system, as well as move the excitation beam in all three dimensions

As a first demonstration of the two-photon functional imaging capabilities of our microscope, we imaged calcium transients from a population of neurons, which provides a window to monitor the action potentials generated by a circuit $^{16,17}$. We accomplished this by selecting targets as described in Section 2.2. First, a conventional raster scanned image of the sample was acquired, and the cell bodies detected. The centers of mass of the somas were used to generate a target map, and a phase mask was generated and sent to the SLM. The laser beam was then split into many beamlets, each one simultaneously targeting a cell body. Total fluorescence was detected with an electron multiplied CCD 
(Hamamatsu, ImageEM) and the image stream analyzed to generate fluorescence time series for each cell. The results are shown in Figure 2. In these preliminary results, we were able to image 50 neurons simultaneously, and at $15 \mathrm{~Hz}$, the $\mathrm{S} / \mathrm{N}$ ratio was still sufficiently high as to be able to reliably detect single action potentials. Signals extracted from the CCD pixels that correspond to the patched neuron and from control neurons were individually analyzed, and demonstrate a reliable correspondence between the evoked action potentials and the measured optical signals ${ }^{4}$. Significantly, we did not notice photobleaching after minutes of exposure, even though the illumination was constant.

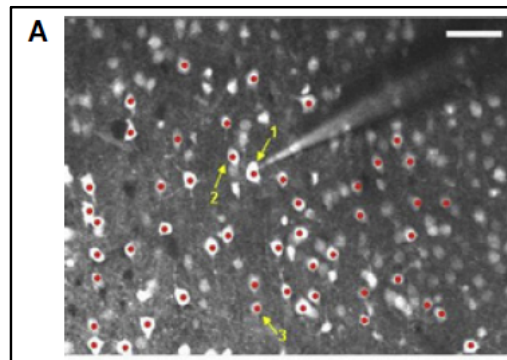

B
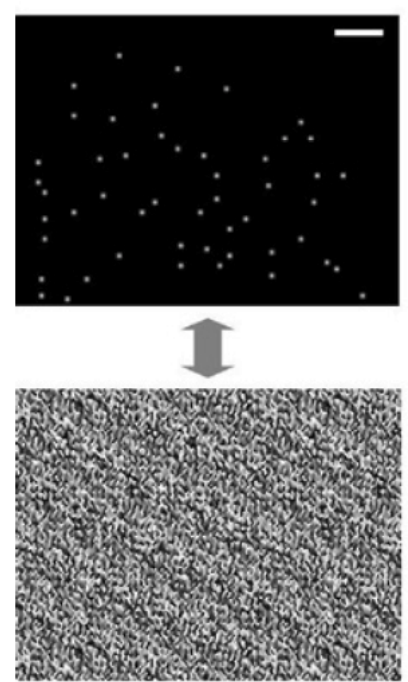

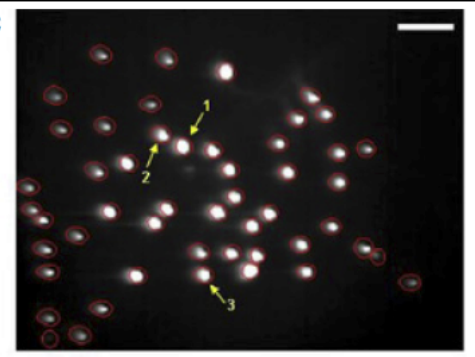

$66 \mathrm{~ms} /$ frame

D

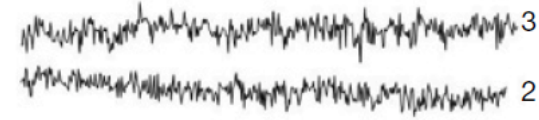

E

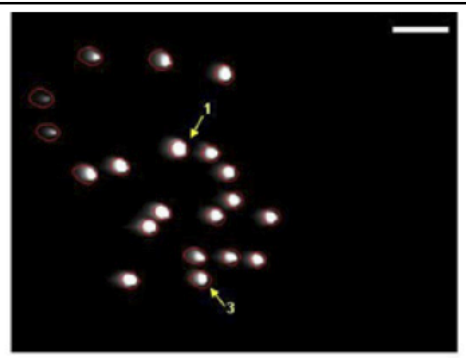

$16 \mathrm{~ms} /$ frame

$\mathbf{F}$

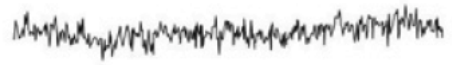
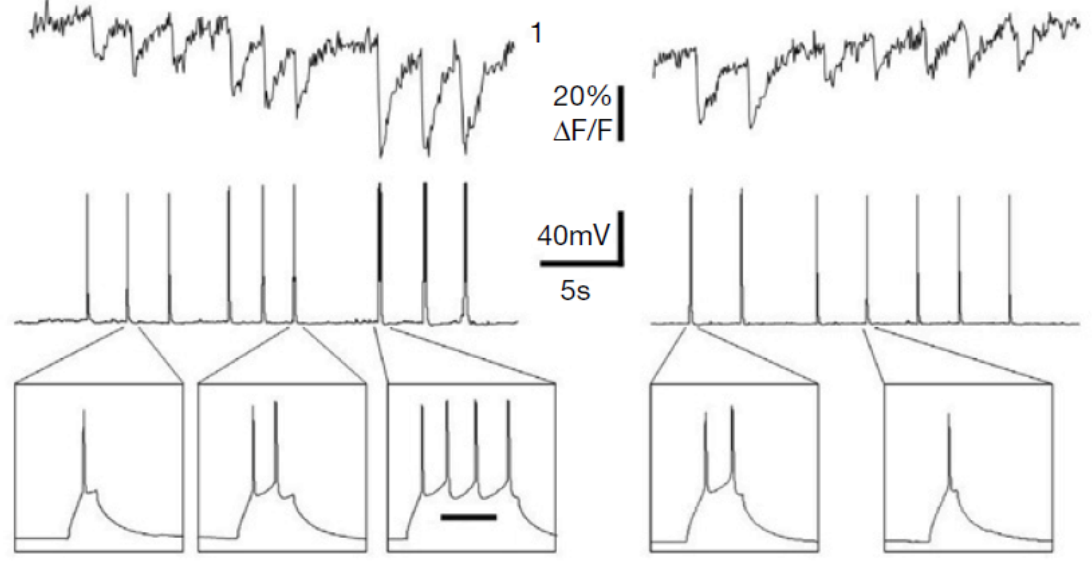

Figure 2. SLM imaging of calcium transients in brain tissue. (A) A neocortical slice (layer 2/3, S1 area, 15 day old mouse) was bulk loaded with a 10:1 mix of Fura-2AM:mag-Indo-1AM). Image shown is a standard raster mode two-photon image. Fifty neurons were targeted for imaging (red spots). The neuron labeled "1" was targeted for patch-clamp recording to trigger action potentials following current injection. The intracellular solution contained $50 \mu \mathrm{M}$ Fura-2 pentapotassium salt, a concentration which roughly corresponds to the intracellular concentration of Fura- 2 achieved by bulk loading (Peterlin et al., 2000). The pipette also contained $10 \mu \mathrm{M}$ Alexa-594 for localization of patched neuron using a different emission filter. (B) Command image file for SLM software and corresponding phase mask. (C) Image of two-photon fluorescence from multiple locations obtained with the camera. Diffractive SLM splits laser beam in order to continuously illuminate spatially different locations with a static pattern $(\sim 4.4 \mathrm{~mW}$ of average excitation power per spot on the sample plane). Red contours were detected using custom software in order to quantify time-lapsed signals from different cells. Notice correspondence between patterns on upper and lower panels. Scale $50 \mu \mathrm{m}$. (D) Calcium signals recorded from stimulated cell (D1) corresponding to different number of elicited action potentials (the panel shows nine current pulses that triggered triplets of 1, 2 and 4 action potentials respectively). Even individual spikes can be detected with good signal-to-noise ratio. Neurons 2 and 3 were not stimulated and do not exhibit change in fluorescent signals. Imaging was performed with $\sim 15$ frames/s temporal resolution $(66 \mathrm{~ms} /$ frame). (E,F) Similar results were obtained with $\sim 60 \mathrm{frames} / \mathrm{s}$ ( $16 \mathrm{~ms} /$ frame), but with higher excitation power per each excitation spot, and less total targets. Seven current pulses were injected, two of them triggered two action potentials, and five triggered individual spikes. No noticeable photobleaching or photodamage was observed over the course of the experiment (several minutes of continuous illumination). Figure taken from Ref 4. 


\subsection{Neuronal control via uncaging}

The basic structure of the RuBi compounds is a ruthenium metal center, with six coordination positions. Four of the sites are occupied by the two bidentate bipyridines, the fifth by an organo-phosphine, and the last by the neuromodulator. A representative structure showing RuBi-glutamate is shown in Figure 3 (A). Upon absorption of one photon of visible light, or two NIR photons, RuBi-glutamate photocleaves within nanoseconds (Figure 3 (B)), and releases free glutamate with an uncaging quantum yield of 0.13 and an effective two-photon cross section of $\sim 0.14 \mathrm{GM}^{18}$.

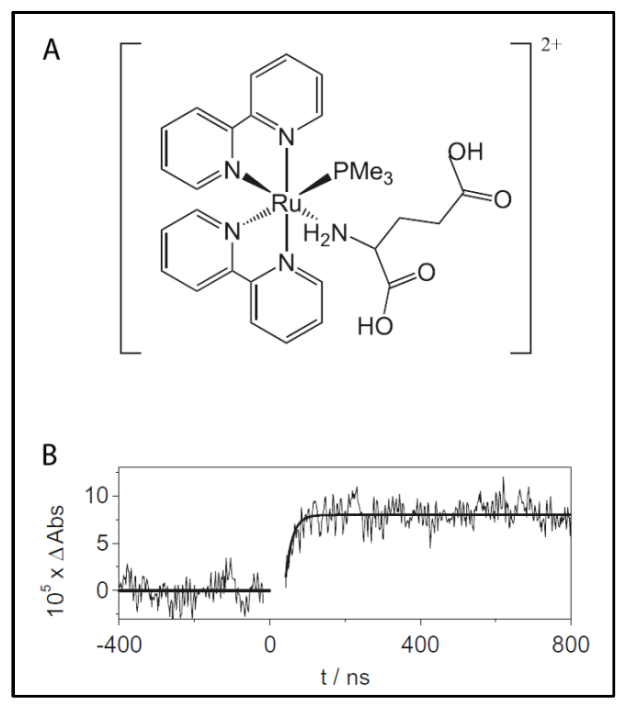

Figure 3 (A) Structure of the protonated form of RuBi-glutamate. (B) The change in absorption of a solution of RuBiglutamate, at $532 \mathrm{~nm}$, corresponding to the release of free glutamate, following flash illumination of RuBi-glut with a high intensity $10 \mathrm{~ns}$ pulse of $532 \mathrm{~nm}$ light.

We used RuBi-glutamate to activate glutamate receptors in layer $2 / 3$ pyramidal neurons while recording the membrane potential. While recording under current clamp, our multi-target protocol was able to reliably generate action potentials (Figure 4, (A) and (B)). Further pharmacological and electrophysiological measurements confirmed the pure glutamatergic activity ${ }^{11}$. In addition to evoking action potentials, weaker stimulations lead to subthreshold depolarizing events. The smallest stimulations, near spines, yielded depolarizations with similar magnitudes and kinetics to physiological synaptic events ${ }^{11}$. Because of the low laser power necessary for the spine stimulations, we were able to use the SLM to stimulate multiple spines simultaneously, and see summation of their inputs ${ }^{4}$. Just as RuBi-glutamate can be used excite the cell, RuBi-GABA can be used to inhibit activity. Our group has previously reported on the use of low concentrations of Rubi-GABA $(<20 \mu \mathrm{M})$ for suppressing action potentials following single-photon excitation at $473 \mathrm{~nm}^{19}$. Using higher concentrations of RuBi-GABA $(\sim 1 \mathrm{mM})$ and two-photon uncaging, we were also able to modulate neuronal activity. With short illumination times, we were able to delay the onset of action potentials produced by direct current injection. With longer illumination times, we were able to completely suppress the firing of the action potential (Figure 4, (C) and (D)).

The RuBi compounds described above, while being effective for stimulation and inhibition, and are not completely innocuous to the system. We note a concentration dependent nonspecific partial block of GABAergic transmission ${ }^{11}$. At low concentrations such as those sufficient for single photon uncaging there are no measurable changes in the system. At the concentrations required for two-photon excitation, we begin to see a diminution in the amplitude of spontaneous GABAergic events, with around a $50 \%$ reduction at concentrations of a $300 \mu \mathrm{M}$ - a concentration high enough for reliable two-photon uncaging ${ }^{11}$. While this is significant, it has not limited the utility of these compound. GABAergic transmission is still sufficiently strong so we are still able to map inhibitory connections with RuBi-glutamate, and with RuBi-GABA, we are able to generate GABA responses of sufficient strength to block cells from firing action potentials, and perform functional mapping of GABA receptors, something that was not possible in our lab using the previous nitrobenyl derivatives ${ }^{11}$. This is not surprising considering that the GABAergic blockade seen when using MNIglutamate, at concentrations sufficient for two-photon uncaging $(>1.5 \mathrm{mM})$, is nearly complete; almost no GABAergic 
transmission can be seen. The exact mechanism of these nonspecific GABAergic effects is not well known for either system, and the groups developing both chemistries are actively working to improve their compounds, and minimize the side effects.

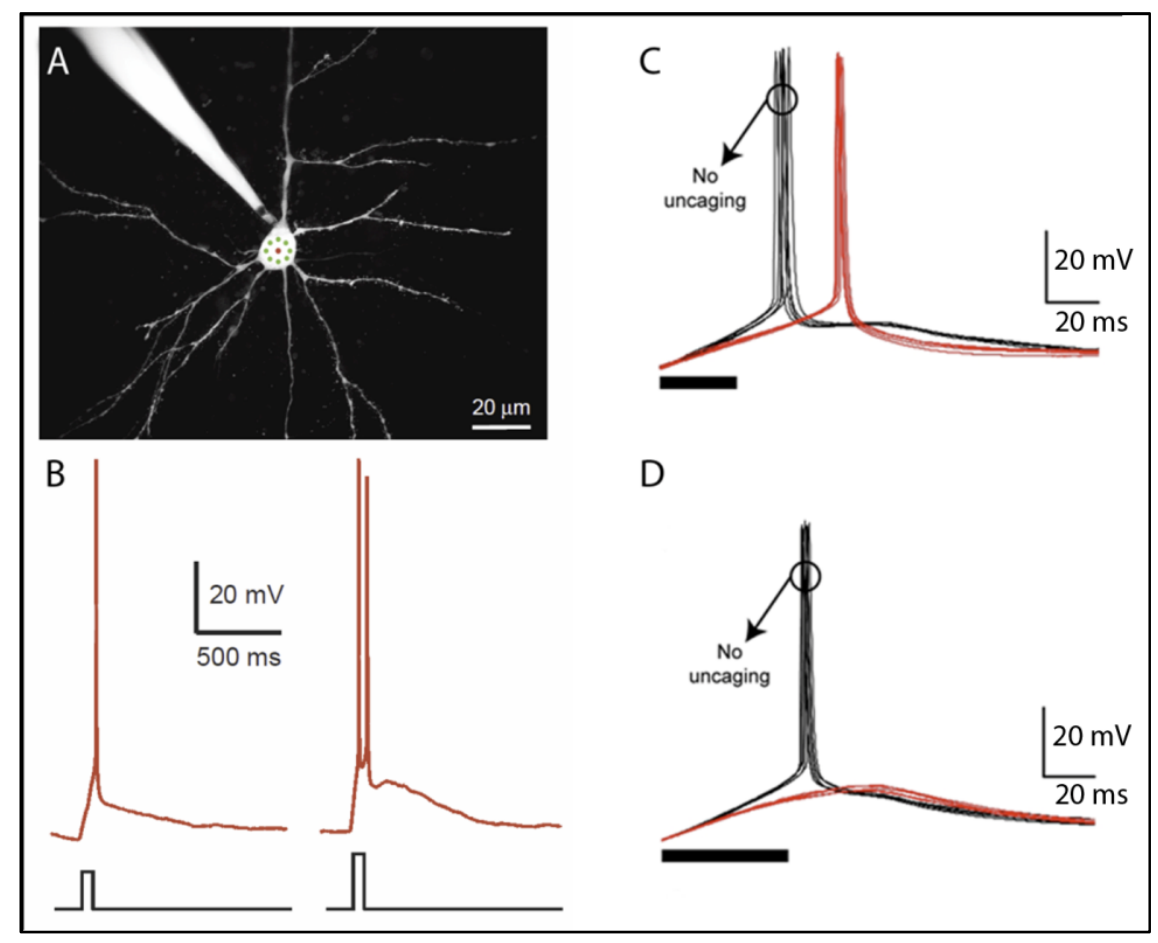

Figure 4 (A) Two-photon image of a layer $2 / 3$ neuron along with the approximate subtarget locations used for RuBiglutamate uncaging. (B) The red traces show the recorded membrane potential in the cell, while the black traces indicate laser power. In the first trace, increasing the laser power uncages glutamate and causes the cell to fire a single action potential. In the second trace, the peak laser power is increased, and the cell fires two action potentials. (C) and (D) RuBiGABA experiments. All traces show the recorded membrane potential of the cell while undergoing a small current injection to evoke action potentials. The black traces indicated trials were the laser was blocked, while the red traces are trials where the laser reaches the sample. The thick black line indicates the time the laser was on. In (C), the laser was on for a short period, uncaging GABA, and delaying the onset of the action potential. In (D), the illumination time was longer, and the action potentials were completely suppressed.

\section{CONCLUSION}

We developed an SLM based microscope for fast two-photon imaging of neuronal activity. Though not limited to nonlinear excitation, the low photoxicity, and increase penetration depth of NIR and IR light, combined with the high spatial resolution, make this scheme particularly attractive. The optical flexibility of the SLM allows patterned illumination to be tailored specifically to the system under study, efficiently redirecting the incoming laser to target only regions of interest. This allows multiple targets to be simultaneously and continuously illuminated, provided a recipe for imaging with high $\mathrm{S} / \mathrm{N}$ and good temporal resolution.

With the SLM providing the means to detect activity, the RuBi compounds allow for the spatially restricted photochemical control of our biological samples. These molecules have high two-photon uncaging cross sections, are readily available, and show lower nonspecific GABAergic blockade than some of the other commonly used two-photon releasable neuromodulators. We have shown that Rubi-glutamate can be used to elicit action potentials, and RuBiGABA can be used to block them, providing control of both excitation and inhibition. Additionally, both compounds can be used to generate physiologically realistic subthreshold responses. We believe the RuBi compounds offer an attractive alternative to the currently available caged compounds, and hope they will allow for detailed studies of neuronal biophysics and circuit properties. Also, because the two-photon cross sections are sufficiently high, and they 
are compatible with the use of an SLM for multiplexed uncaging, we plan to use them to explore dendritic integration. Specifically, the activation of several dendritic spines in any arbitrary spatio-temporal pattern appears as an ideal experimental approach. Moreover, the near synchronous activation of multiple cells could be an important requirement for the engagement of cortical circuits and the ability to simultaneously activate arbitrary groups of neurons has the potential to significantly aid the burgeoning field of circuit neuroscience. Therefore, we think it is likely that the SLM microscope and the RuBi compounds will be of great utility in circuit neuroscience.

\section{ACKNOWLEDGEMENTS}

We thank Emiliano Rial Verde for his contributions to the two-photon uncaging experiments with RuBi-GABA.

\section{REFERENCES}

[1] Denk, W., Strickler, J. H., and Webb, W. W., "Two-photon laser scanning fluorescence microscopy”, Science 248, 73-76 (1990)

[2] Hopt, A., and Neher, E., "Highly nonlinear photodamage in two-photon fluorescence microscopy", Biophys. J. 80, 2029-2036 (2001)

[3] Koester, H. J., Baur, D., Uhl, R., and Hell, S. W., "Ca2+ fluorescence imaging with pico- and femtosecond twophoton excitation: signal and photodamage”, Biophys. J. 77, 2226-2236 (1999)

[4] Nikolenko, V., Watson, B. O., Araya, R., Woodruff, A., Peterka, D. S., and Yuste, R., "SLM Microscopy: scanless two-photon imaging and photostimulation using spatial light modulators", Front. Neural. Circuits 2-5, 1-14 (2008)

[5] Ellis-Davies, G. C., [Basics of photoactivation. In Imaging in Neuroscience and Development: A Laboratory Manual], R. Yuste and A. Konnerth, eds, Cold Spring Harbor Press, Cold Spring Harbor, New York, pp. 367-373 (2005)

[6] Matsuzaki, M., Ellis-Davies, G. C., Nemoto, T., Miyashita, Y., Iino, M., and Kasai, H., "Dendritic spine geometry is critical for AMPA receptor expression in hippocampal CA1 pyramidal neurons", Nat. Neurosci. 4, 1086-1092 (2001)

[7] Araya, R., Jiang, J., Eisenthal, K. B., and Yuste, R., "The spine neck filters membrane potentials", Proc. Natl. Acad. Sci. U.S.A. 103, 17961-17966 (2006)

[8] Carter, A. G., and Sabatini, B. L., "State-dependent calcium signaling in dendritic spines of striatal medium spiny neurons", Neuron 44, 483-493 (2004)

[9] Sobczyk, A., Scheuss, V., and Svoboda, K., "NMDA receptor subunit-dependent Ca2+ $\downarrow$ signaling in individual hippocampal dendritic spines", J. Neurosci. 25, 6037-6046 (2005)

[10] Nikolenko, V., Poskanzer, K. E., and Yuste, R., "Two-photon photostimulation and imaging of neural circuits", Nat. Methods 4, 943-950 (2007)

[11]Fino, E., Araya, R., Peterka, D.S., Salierno, M., Etchenique, R., Yuste, R., "RuBi-Glutamate: Two-photon and visible-light photoactivation of neurons and dendritic spines", Front. in Neural Circuits, 3 (2009)

[12]Zayat, L., Calero, C., Albores, P., Baraldo, L., and Etchenique, R., "A new strategy for neurochemical photodelivery: metal-ligand heterolytic cleavage." J. Am. Chem. Soc. 125, 882-883 (2003)

[13] Chartier, G., [Introduction to Optics], Springer Science, New York, NY (2005)

[14]Fienup, J. R., and Wackerman, C. C., "Phase-retrieval stagnation problems and solutions", J. Opt. Soc. Am. A 3, 1897-1907 (1986)

[15] Bauschke, H., Combettes, P. L., and Luke, D. R., "Phase retrieval, error reduction algorithm, and Fienup variants: a view from convex optimization", J. Opt. Soc. Am. A 19, 1334-1345 (2002)

[16] Yuste, R., and Katz, L. C., "Control of postsynaptic Ca2+ influx in developing neocortex by excitatory and inhibitory neurotransmitters", Neuron 6, 333-344 (1991)

[17] Lillis, K. P., Eng, A., White, J. A., and Mertz, J., "Two-photon imaging of spatially extended neuronal network dynamics with high temporal resolution", J. Neurosci. Methods 172, 178-184 (2008)

[18] Salierno, M., Marceca, E., Peterka, D.S., Yuste, R., Etchenique, R., "A fast ruthenium polypyridine cage complex photoreleases glutamate with visible or IR light in one and two photon regimes", J. Inorg. Biochem., doi:10.1016/j.jinorgbio.2009.12.004 (in press) (2010) 
[19] Rial Verde, E.M., Zayat, L., Etchenique, R., Yuste, R., "Photorelease of GABA with visible light using an inorganic caging group", Front. in Neural Circuits, 2 (2008) 